\title{
An Improved Method of Removing Fog and Haze Effect From Images
}

\author{
Jia SHI ${ }^{1, a}$, Ke-jian $Y_{A N G}{ }^{2, b}$ \\ Wuhan University of Technology, WuHan,China \\ a838357982@qq.com, bykjcgs@whut.edu.cn
}

Keywords: Image dehazing, Histogram equalization,Blocking effect, Interpolation

\begin{abstract}
To process the fog and haze image,a local histogram equalization based on distant scale factor interpolation method to deal with the blocking effect was proposed .In this paper,We analyzed the basic features of the haze image,that is, low brightness and low contrast. And then,for these features we choose local histogram equalization and proposed an improved interpolation method to eliminate the blocking effect.The simulation results demonstrated that the proposed method can reduce time consumption,while suppressing the color distortion,improving the image contrast and getting satisfactory results.
\end{abstract}

\section{Introduction}

In fog and haze weather, scattering and absorption of atmospheric aerosols could result in images captured by the image sensors appearing low contrast, blur, and color distortion. Image dehazing can effectively enhance the contrast, improve the visual effect, and remove impacts of bad weather on the image quality. Therefore, the research of image dehazing methods has greatly theoretical and practical significance.

Recently,significant progress has been made in single image dehazing.Generally,the image dehazing technique can be roughly divided into two categories based on whether or not using the atmospheric scattering model:One is based on non-physical model of the image dehazing algorithm,this kind of methods does not consider the degradation process of fog and haze image, only enhances the haze image by using the existing image processing algorithm,such as histogram equalization[1][2] and gamma transformation methods[3]; the other is based on physical model of image dehazing algorithm,this method analyzes the interaction between particles in the air and the atmosphere, establishes the atmospheric scattering model, estimates the parameters in the model and inverses the true scene image without haze,such as Tan and He proposed methods[4-6].

The haze images generally show a low brightness and low contrast features.So we can use the image enhancement method to deal with haze images.Local histogram equalization method is more suitable for image dehazing processing.This method divides image into a series of sub-blocks, and then using histogram equalization for each block. But This method will produce blocking effect which will affect the visual effect of the picture. Zuiderveld proposed CLAHE method use the bi-linear interpolation to eliminate blocking effect, achieved good results,but there is distortion in the processed image[7].WANG proposed an improved interpolation method to deal with blocking effect,but its too complex to use in reality[8]. In this paper, we propose a distance-based interpolation method to deal with blocking effect,that not only improves the efficiency of algorithm implementation, but also reduces color distortion.

The rest of this paper is organized as follows:Section II is to describe our method,Section III is to show the the simulation and comparison results,And section IV is to conclude this whole paper. 


\section{Proposed Methodology}

In traditional histogram equalization[9-10],the mapping function is the cumulative distribution function of whole input image,so the processed image may lose a lot of details. The local histogram equalization proposed to improved traditional histogram equalization method,but the processed image exist obvious block effect.

In the image processing, for each pixel p inside the image, four adjacent pixels in the horizontal and vertical directions are called 4-neighborhood of $\mathrm{p}$, the adjacent four corner pixels and the 4-neighborhood pixels called 8-neighborhood of p.As shown in Fig. 2, it is generalized to the case of sub-blocks, in which the darkest color is the center sub-block, the lighter sub-block is the 4-neighborhood sub-block, and the four diagonally adjacent sub-blocks are 8-neighborhood sub-blocks.

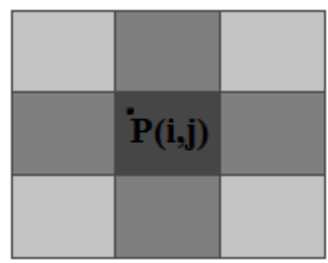

Figure 1. 4-neighborhood and 8-neighborhood

In Fig.1,when process pixel p,first of all,we should consider the influence of its own sub-block (center sub-block) on its gray-scale mapping. Secondly, we should consider 4-neighborhood sub-block, which are directly adjacent to it, and finally the remaining 4 corners the 8-neighborhood sub-block should be taken into account. Based on the above analysis, we propose an interpolation method based on distance scale factor, and extend this interpolation method to 4-neighborhood and 8-neighborhood. In order to reduce the computational complexity, we use the block distance to measure the proportion of interpolation factor, in this way, the efficiency of the algorithm will be multiplied.The formula for calculating the block distance between any two points $P_{a}\left(x_{1}, y_{1}\right)$ and $P_{b}\left(x_{2}, y_{2}\right)$ in a two-dimensional plane space can be expressed as:

$$
d_{a, b}=\left|x_{1}-x_{2}\right|+\left|y_{1}-y_{2}\right|
$$

As Eq.1 show ,compared to the Euclidean distance,the block distance solving process is indeed fast.

Fig.2 is a schematic diagram of the improved interpolation method,when process the pixel $P(i, j)$,pixel $P_{2}, P_{4}, P_{6}, P_{8}, P_{9}$ is the center point of the 4-neighborhood sub-block,and the corresponding mapping function is $\left(T_{2}, T_{4}, T_{6}, T_{8}, T_{9}\right)$.Calculate the block distance $d_{2}, d_{4}, d_{6}, d_{8}, d_{9}$ between $P(i, j)$ and $P_{2}, P_{4}, P_{6}, P_{8}, P_{9}$ according to Eq. 2.Since the distance is inversely proportional to the influence on the sub-block,so we should convert the distances.Assuming the size of sub-block is $m \times n$,then the distance from the center of 4-neiborhood sub-block to any point in the center block is less than $\frac{3}{2}(m+n)$,so define the conversion formula as:

$\bar{d}=\frac{3}{2}(m+n)-d$

The converted distance is $\overline{d_{2}}, \overline{d_{4}}, \overline{d_{6}}, \overline{d_{8}}, \overline{d_{9}}$, after this transformation ,the impact on point 
$P(i, j)$ becomes proportional relationship,that is,the larger the converted distance , the greater the influence on the center sub-block, and vice versa. In summary, the output gray value of the $P(i, j)$ can be interpolated according to the Eq. 2:

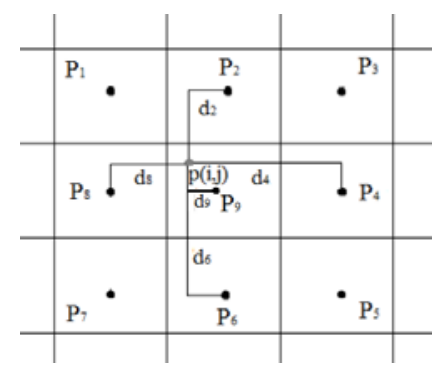

Figure 2. The graph of improved interpolation method

$T(i, j)=w_{2} T_{2}(i, j)+w_{4} T_{4}(i, j)+w_{6} T_{6}(i, j)+w_{8} T_{8}(i, j)+w_{9} T_{9}(i, j)$

Where,

$w_{2}, w_{4}, w_{6}, w_{8}, w_{9}$ are the distance scale factor which are the ratios of the converted distance and the sum of the converted distances, that is $w_{2}=\frac{\overline{d_{2}}}{\text { sum }}, w_{4}=\frac{\overline{d_{4}}}{\text { sum }}, w_{6}=\frac{\overline{d_{6}}}{\text { sum }}$, $w_{8}=\frac{\overline{d_{8}}}{\text { sum }}, w_{9}=\frac{\overline{d_{9}}}{\text { sum }}$, sum $=\overline{d_{2}}+\overline{d_{4}}+\overline{d_{6}}+\overline{d_{8}}+\overline{d_{9}}$

As shown in Fig. 3 ,In particular,the four dark gray corner sub-block and light gray border sub-block do not exist completely 4-neighborhood sub-block, such as sub-block Adoes not exist two sub-blocks on the left side and the top side, and the 4-neighborhood sub-block are missing half. For this special case, the sub-block that can not be considered and have no effect on the gray mapping of the central sub-block, that is ,can be neglected. The corresponding scaling factor can be set to zero. For the four corner sub-blocks, only the three nearest neighbor sub-block are considered, and the algorithm degenerates into bi-linear interpolation method. For the boundary sub-blocks ,only one sub-block is missing.

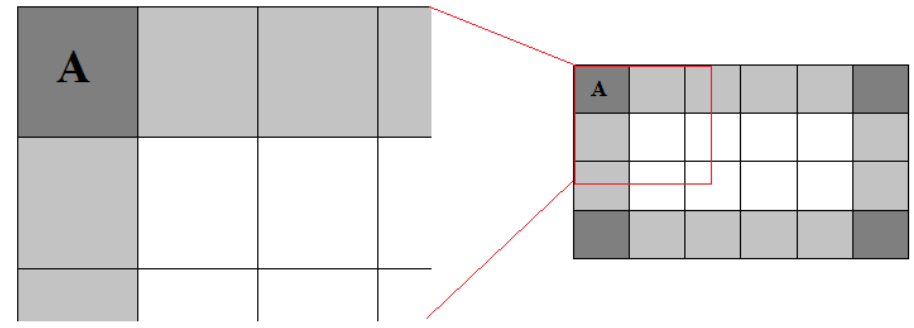

Figure 3. Boundary conditions of sub-block

\section{Simulation and comparsion}

In this section,we implement four typical methods using matlab 2013 and compare the experiment results from subjective evaluation and objective evaluation.All experiments are run on a $3.1 \mathrm{~Hz}$ Intel Core i3 CPU with $4 \mathrm{~GB}$ ram.

The proposed method can be divided into the following steps: 
Step 1: Divide the input image into a series of sub-blocks;

Step 2: Calculate the mapping function for each sub-block;

Step 3: Traverse each pixel from top to bottom from left to right;

Step 4:Determine whether the sub-block is corner or boundary sub-blocks,Y goto

\section{Step 6,N goto Step 5;}

Step 5:According to the sub-block is missing, modify the corresponding scale factor to 0 ;

Step 6:According to the Eq.3, calculate the output gray value of the processing pixel;

Step 7:Repeat Step 3-6 until the traversal is complete.

We selected 20 fog and haze images to carry out the experiment,and all the origin images in this paper come from HE's and Tan's paper.By comparing the results of four different method ,we can analysis the performance of the corresponding algorithm.

Fig. 4 is the experiment of cannon3.bmp, Fig 4(a) is the origin fog and haze image of low contrast and low brightness.Fig 4(b) is the result of HE method processing,the sky is over enhanced but the leaves' contrast did not stretch and the outline and details of the building were lost.Fig 4(c) is the result of bi-linear interpolation method processing,the color of the leaves is a bit distorted.Fig 4(d) is the result of WANG's method processing, the sky is also over enhanced and the overall color of the image is bright.Fig 4(e) is the result of our method processed,the whole image is properly enhanced,the color of leaves turns green and color distortion is suppressed,the part of sky and distant buildings clearly distinguished.

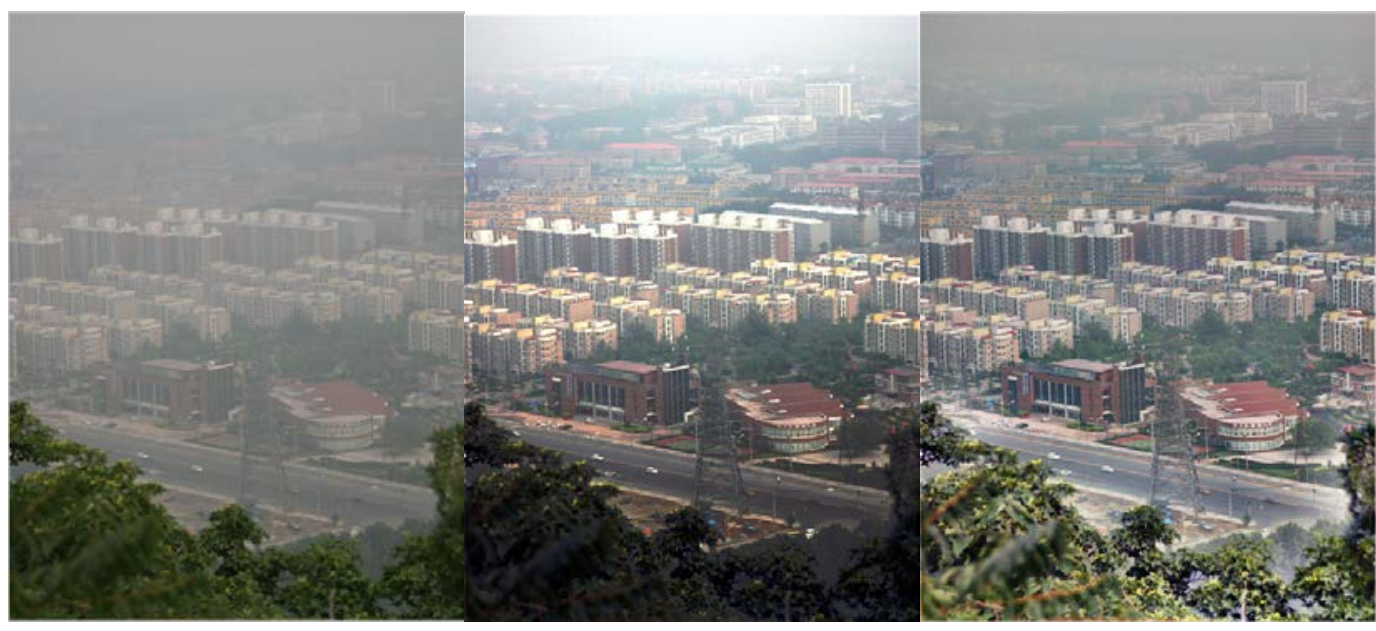

(a) origin image

(b) HE method

(c) bi-linear interpolation

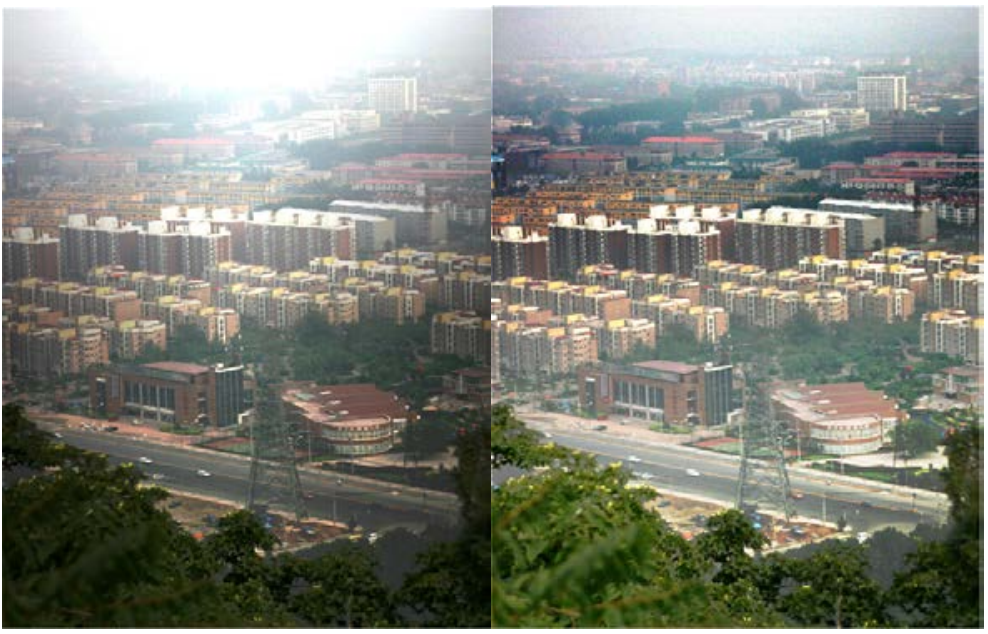

(d) WANG's method

(e) our method

Figure 4. The experiment of cannon3.bmp 
For time efficiency analysis,we choose 10 fog and haze images of size $600 * 400$ pixels to do experiment,the performance of the algorithm is compared by calculating the average time of each algorithm.

Table 1 . The average execution time of four methods

\begin{tabular}{cc}
\hline Method & Time (second) \\
\hline Histogram equalization & 0.107204 \\
Bi-linear interpolation & 0.302381 \\
Area scale factor interpolation & 0.430493 \\
Our Method & 0.278452 \\
\hline
\end{tabular}

From Table 1,The traditional Histogram equalization method is more effective,only using 0.107204 seconds.In the remaining three interpolation algorithms,our method and Bi-linear interpolation are more effective,the execution time of the algorithm is about 0.3 seconds.

For these three interpolation(bi-linear interpolation,area scale factor and our method) methods, we count the times of multiplication and division operations required for each interpolation, according to the required multiplication and division operations to measure the time of algorithm implementation.The detailed statistics are shown in Table 2.

Table 2. The multiplication and division times of three interpolation

\begin{tabular}{|c|c|c|c|c|}
\hline Method & Multiplication & division & Total times & description \\
\hline $\begin{array}{l}\text { Bi-linear } \\
\text { interpolation }\end{array}$ & 8 & 2 & 10 & $\begin{array}{c}\text { Interpolation:8 times; Normalized: } 2 \\
\text { times }\end{array}$ \\
\hline $\begin{array}{l}\text { Area scale factor } \\
\text { interpolation }\end{array}$ & 8 & 4 & 12 & $\begin{array}{c}\text { Interpolation:4times; Area :8 times; } \\
\text { Factor:4 times }\end{array}$ \\
\hline Our method & 5 & 5 & 10 & Interpolation:5times:Factor:5times \\
\hline
\end{tabular}

From Table 2, we can see that the relationship among the times of multiplication and division of the four interpolation algorithms: our method (10 times) = Bi-linear interpolation(10 times) < Area scale factor Method (12 times).So our method is the same as bi-linear interpolation algorithm in execution time.

\section{Summary}

Image dehazing technology is a very challenging task,local histogram equalization is a more suitable method of image dehazing,but this kind of method will divide the input image into a series of sub-blocks before processing ,so the processed image exist blocking effect. In this paper we proposed an improved interpolation method based on distance scale factor to deal with the blocking effect.The experimental results show our method is more effective than other method,and the contrast of the processed pictures is enhanced,and eliminate the effect of fog and haze basically.

But this methods also have some weak points,the number of sub-blocks is closely related to the quality of processed image.When the number of sub-blocks is large,the processed image will over enhanced,but when the number of of sub-blocks is small,this method degrades to traditional histogram equalization.So our future work will focus on the relationship between the number of 
sub-blocks and the quality of processed image.

\section{References}

[1] Pizer, Stephen M., et al. "Adaptive histogram equalization and its variations." Computer Vision Graphics \& Image Processing39.3(1987):355-368.

[2] Kim, Joung Youn, L. S. Kim, and S. H. Hwang. "An advanced contrast enhancement using partially overlapped sub-block histogram equalization." IEEE Transactions on Circuits \& Systems for Video Technology 11.4(2001):475-484.

[3] Zhang, L., and Z. D. Zhu. "A novel nonlinear method for image enhancement based on nonsubsampled contourlet transform." Journal of Electronics \& Information Technology 31.8(2009):1786-1790.

[4] Tan, Robby T. "Visibility in bad weather from a single image." (2008):1-8.

[5] He, K., J. Sun, and X. Tang. "Single Image Haze Removal Using Dark Channel Prior. " IEEE Transactions on Pattern Analysis \& Machine Intelligence 33.12(2010):2341-2353.

[6] Fattal, Raanan. "Single image dehazing." Acm Transactions on Graphics27.3(2008):1--9.

[7] Zuiderveld, Karel. "Contrast Limited Adaptive Histogram Equalization."Graphics Gems (1994):474-485.

[8] Wang, Ping, C. Zhang, and Y. X. Luo. "Fast algorithm to enhance contrast of fog-degraded images." Journal of Computer Applications26.1(2006):152-151.

[9] Digital Image Processing. Springer London, 2008.

[10] Gonzalez, Rafael C., R. E. Woods, and S. L. Eddins. Digital image processing using MATLAB. Digital image processing /. Pearson/Prentice Hall, 2007:197-199. 